\title{
Head and Neck Cancer During Covid-19 Pandemic: Was there a Diagnostic Delay?
}

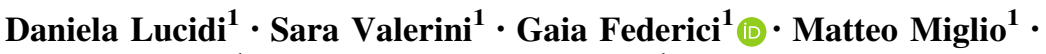 \\ Carla Cantaffa $^{1} \cdot$ Matteo Alicandri-Ciufelli $^{1}$
}

Received: 14 September 2021 / Accepted: 27 December 2021/Published online: 15 January 2022

(C) Association of Otolaryngologists of India 2022

\begin{abstract}
The aim of the present study is to investigate a possible delay in diagnosis and therapy administration for Head and Neck oncological patients, during the Sars-Cov2 pandemic and to compare the median tumor stage (MTS) at presentation. This is a retrospective review on patients who presented at the University Hospital of Modena with a newly diagnosis of Head and Neck cancer (HNC), comparing the first pandemic period (from March 1st, to October 15th 2020) to the same period of 2019. The time in days from the diagnostic suspicion to the beginning of the treatment, hereafter referred to as delay in treatment initiation (DTI), and the tumor stage were calculated for all the enrolled patients. Mean percentage of reduction of the clinical and surgical activities was $-25.8 \%$ (range: 66-1.5\%), with higher percentages in phase 1 for all the analyzed activities. $125 \mathrm{HNC}$ patients were enrolled in the analysis as the study group, compared with a cohort of 140 patients from the previous year. The MTS was III for both groups, however a significant statistical difference was determined in the stage comparison, $p=0.023$. When DTI was analyzed no statistically significant differences were appreciated. A more advanced stage at presentation was demonstrated at our institution for HNC patients during the first phase of the Covid-19 pandemic in 2020. However, a statistically significant delay in time between diagnosis and therapy administration was not assessed, thus confirming the maintenance of a high level of care even in the COVID19 era.
\end{abstract}

Gaia Federici

gaia.federici@unimore.it

1 Otolaryngology and Head and Neck Surgery Department, University Hospital of Modena, Via del Pozzo 71, 41125 Modena, Italy
Keywords Head and Neck cancer - Delayed diagnosis · SARS-CoV-2 $\cdot$ Health care systems $\cdot$ Pandemics

\section{Introduction}

The COVID-19 pandemic has placed increased stress on health care systems during 2020. As a result, the management of cancer patients has suffered critical resource shortages and delays in care [1]. Treatment for Head and Neck Cancer (HNC) was among those most affected by the detrimental effects of the pandemic for many reasons [2]. Otolaryngologists around the world have been forced to make changes in their practice as evidence emerged that they are at significantly higher risk of infection, due to exposure to the upper airway and respiratory droplets [3-6]. Poor evidence-based data regarding HNC management were available during the pandemics to base decisions upon. In the early phases of the pandemic the American College of Surgeons has recommended postponing deferrable surgery, including that for low-risk cancers [7]. Similarly, the American Academy of Otolaryngology-Head \& Neck Surgery has recommended surgeons to delay elective cases in order to allow for improved safety of staff and patients and to expand hospital capacity for more emergent conditions during the pandemic [8]. Unfortunately, the pandemic has lasted for a long time now, and some aspects of HNC management need to be reviewed. Postponing treatments can adversely affect HNC mortality rates [9]; it is a time-dependent disease and when only best supportive care is employed, nearly half of patients will die of cancer progression within months [10]. 
We hypothesized that, following an initial phase of health services massive reduction, a delay in HNC diagnosis and an increase in the time to initiation of curative treatment might have happened.

The aim of the present study was (1) To define three definite time periods during the 2020 pandemic, characterized by different health services supply at our institution; (2) To compare the median tumor stage of HNC patients evaluated at the HNC-MT consultations in the different time periods versus similar periods of the previous year; (3) To analyze the time elapsed between the first diagnostic suspicion and the initiation of treatment, and to compare it with a similar period of the previous year, to investigate a possible delay in therapy administration.

\section{Materials and Methods}

This is a retrospective review on HNC patients who presented at the University Hospital of Modena, Italy, over a period of 228 days from March 1st, 2020 to October 15th, 2020. This period was compared to the analogous period a year before, from March 1st, 2019 to October 15th, 2019.

\section{Definition of Time Periods for Analysis}

Three time periods during the 2020 pandemic were chosen, to coincide with different phases: phase 1 (from March 1st, 2020 to May 15th, 2020) when a severe reduction of the clinical and surgical activities was observed, due to the first massive pandemic spike; phase 2 (from May 16th, 2020 to July 31st, 2020) or graduated re-opening; phase 3 (from August 1st, 2020 to October 15th, 2020) or complete reopening and resumption of activities at full capacity. The number of 1 st level outpatients' visits (regardless of the referral cause), the number of patients referring to $\mathrm{HNC}$ multidisciplinary team or HNC-MT consultations (including both first and follow-up visits) and the number of overall performed surgeries were compared among the 3 periods to confirm the hypothesized differences in the clinical activities.

\section{Comparison of the Disease Stage}

Patients presenting during the previously defined 2020 periods with newly diagnosed laryngeal, salivary, skin of the head and neck, nasal, paranasal, oral, pharyngeal and occult cancer were enrolled as the study group. Patients with benign neoplasms, patients with recurrent cancer who were already in follow-up protocols at the HNC-MT consultation, patients with cancer of other subsites (i.e. thyroid) and patients whose clinical data were not fully available were excluded from the study. Patients presenting during the same period of the previous year were enrolled, based on analogous criteria, as the control group. Disease stage, defined as clinical TNM (cTNM), was assessed at the first HNC-MT consultation, according to the AJCC 8th edition TNM staging (from early [I-II] to advanced [III-IV] tumor stage). cTNM was obtained for all patients and median stages for each tumor subsite were compared with those from the control group. Patients were also categorized according to the different therapeutic approach, and comparisons were made between groups.

\section{Delay in Treatment Initiation}

Time, expressed in days, from the diagnostic suspicion to the beginning of the treatment (intended as the date of surgery or the first day of chemotherapy/radiotherapy administration), hereafter referred to as Delay in Treatment Initiation (or DTI), was calculated for all the enrolled patients. The time of diagnostic suspicion was defined as the first specialist visit when the hypothesis of a tumor was formulated and the diagnostic process was initiated. Mean DTI time, stratified by pandemic phase, was compared with that from the same period one year before. In case of diagnostic pathways that extended between two periods, the patient was included in the study group corresponding to the period in which the diagnostic suspicion occurred.

Descriptive statistical analysis, such as medians and ranges were used as appropriate. Pearson's Chi square and Fisher's exact tests were used to examine associations between groups for categorical variables and the MannWhitney $\mathrm{U}$ test was used for continuous variables. A $p$ value of less than 0.05 was considered significant. The statistical analysis of the results was performed using SPSS for Windows (IBM SPSS Statistics, Chicago, USA). The study involved the auditing of retrospective data already available on our hospital patient management system. As the data reported by the clinicians in the clinical notes did not deviate from standard practice, no special approval was sought for the study.

\section{Results}

\section{Definition of Time Periods for Analysis}

Table 1 describes the number of performed surgeries, number of patients who referred to 1st level outpatients' visits and number of patients who referred to HNC-MT consultations (including both first and follow-up visits), divided for the 3 phases in 2020 e 2019 . Mean percentage of reduction of the clinical and surgical activities was $25.8 \%$ (range: $-66-1.5 \%$ ), with higher percentages in phase 1 for all the analyzed activities. 
Table 1 clinical and surgical activities performed during the 3 phases in 2019 and 2020. HNC-MDT = Head and Neck Cancer-Multi Disciplinary Team

\begin{tabular}{|c|c|c|c|}
\hline Performed surgeries (n.) & $\begin{array}{l}3 / 1-5 / 15 \\
\text { (phase 1) }\end{array}$ & $\begin{array}{l}5 / 16-7 / 31 \\
\text { (phase 2) }\end{array}$ & $\begin{array}{l}8 / 1-10 / 15 \\
\text { (phase 3) }\end{array}$ \\
\hline 2019 & 374 & 345 & 271 \\
\hline 2020 & 127 & 294 & 273 \\
\hline Reduction percentage & $-66.04 \%$ & $-14.8 \%$ & $0.7 \%$ \\
\hline \multicolumn{4}{|c|}{ Patients referred to outpatient's visits (n.) } \\
\hline 2019 & 3856 & 3092 & 2958 \\
\hline 2020 & 1394 & 2383 & 2306 \\
\hline Reduction percentage & $-63.8 \%$ & $-22.9 \%$ & $-22 \%$ \\
\hline \multicolumn{4}{|c|}{ Patients referred to HNC-MDT consultations (n.) } \\
\hline 2019 & 287 & 319 & 259 \\
\hline 2020 & 179 & 297 & 263 \\
\hline Reduction percentage & $-37.6 \%$ & $-6.9 \%$ & $1.5 \%$ \\
\hline
\end{tabular}

\section{Comparison of the Disease Stage}

$125 \mathrm{HNC}$ patients were enrolled in the analysis as the study group. A cohort of 140 patients who presented during the corresponding 2019 period was included as the control group. Distribution of the percentages of patients' referral over the 3 phases in 2020 and 2019 is displayed in Fig. 1; the differences between groups were not statistically significant ( $p>0.05$ for both comparisons). Mean age in the 2019 group was 68.5 years (range: $25-96 ; \pm 12.2$ ) versus 66.4 years (range: $21-91 ; \pm 13.3$ ) in the 2020 group $(p>0.05)$. The tumor characteristics are displayed in Table 2. Differences in the distribution of primary tumor subsites between groups were not statistically significant $(p>0.05)$. Table 2 illustrates the differences in tumor staging between 2019 and 2020. The median tumor stage was III for both groups, however a significant statistical difference was determined in the stage comparison ( $p=0.023$, Fig. 2). When stage IV patients were further stratified by stage IVa, IVb and IVc no significant difference was determined in the inter-group comparison $(p>0.05)$. By analyzing the tumor stage among the three phases no significant differences were found both in 2019 and 2020 ( $p>0.05$ for both analysis). Table 3 depicts the analysis of the different therapeutic approaches performed in the two groups; no statistically significant difference was determined comparing the two groups $(p>0.05)$.

\section{Delay in Treatment Initiation}

The DTI was 47.6 days (range: $10-150 ; \pm 32$ ) in 2019 group versus 44 days (range: $8-151 ; \pm 30.3$ ) in 2020 group. The difference was not statistically significant $(p>0.05)$. When DTI was analyzed for the 3 different
Fig. 1 distribution of the percentages of patients' referral over the 3 phases in 2020 e 2019

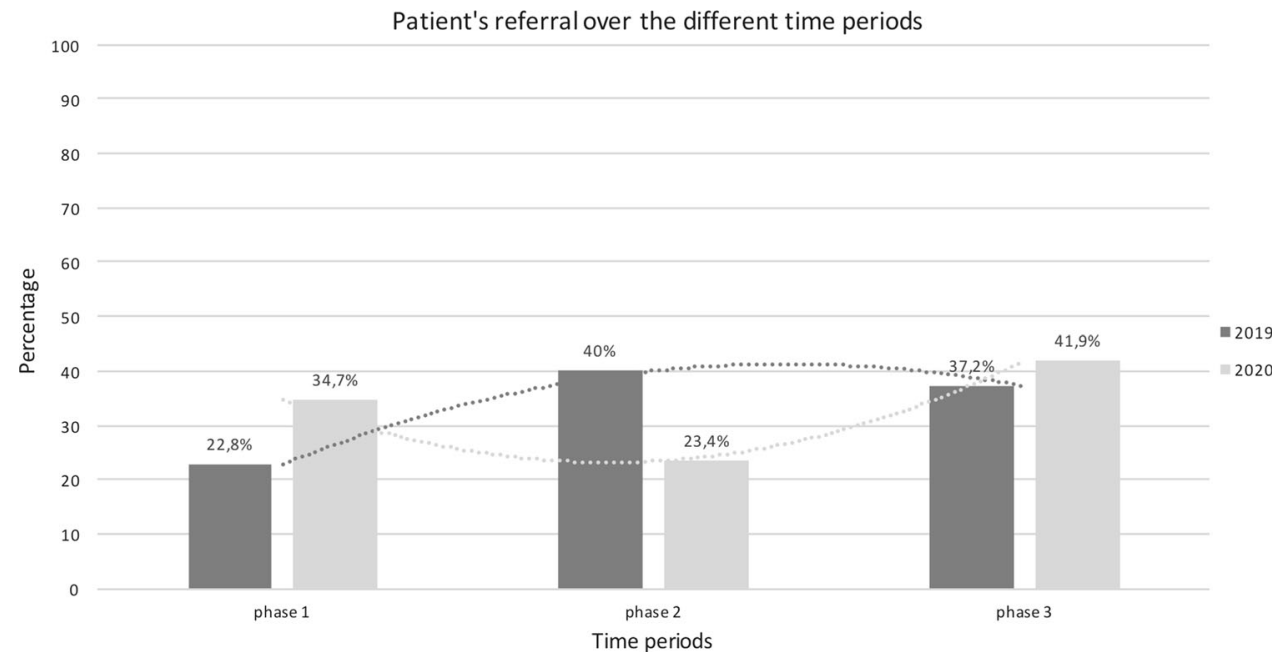


Table 2 tumor characteristics

\begin{tabular}{|c|c|c|c|c|}
\hline \multirow{2}{*}{$\frac{\text { Year }}{2019}$} & \multicolumn{2}{|l|}{ Primary tumor subsite $(\mathrm{n} ; \%)$} & \multicolumn{2}{|c|}{ Tumor staging (n; \%) } \\
\hline & Nose and paranasal cavities & $12-8.6 \%$ & I & $43-30.7 \%$ \\
\hline & Oral cavity & $24-17.1 \%$ & II & $19-13.5 \%$ \\
\hline & Skin of the Head and Neck & $16-11.4 \%$ & III & $30-21.4 \%$ \\
\hline & Pharynx & $30-21.5 \%$ & IV (overall) & $48-34.4 \%$ \\
\hline & Salivary Glands & $1-0.7 \%$ & IVa & 28 \\
\hline & Larynx & $56-40 \%$ & $\mathrm{IVb}$ & 16 \\
\hline & Occult & $1-0.7 \%$ & IVc & 4 \\
\hline \multirow[t]{7}{*}{2020} & Nose and paranasal cavities & $13-0.4 \%$ & I & $31-24.8 \%$ \\
\hline & Oral cavity & $20-16 \%$ & II & $16-12.8 \%$ \\
\hline & Skin of the Head and Neck & $13-10.4 \%$ & III & $17-13.6 \%$ \\
\hline & Pharynx & $28-22.4 \%$ & IV (overall) & $61-48.8 \%$ \\
\hline & Salivary Glands & $5-4 \%$ & IVa & 35 \\
\hline & Larynx & $44-35.2 \%$ & $\mathrm{IVb}$ & 20 \\
\hline & Occult & $2-1.6 \%$ & IVc & 6 \\
\hline
\end{tabular}

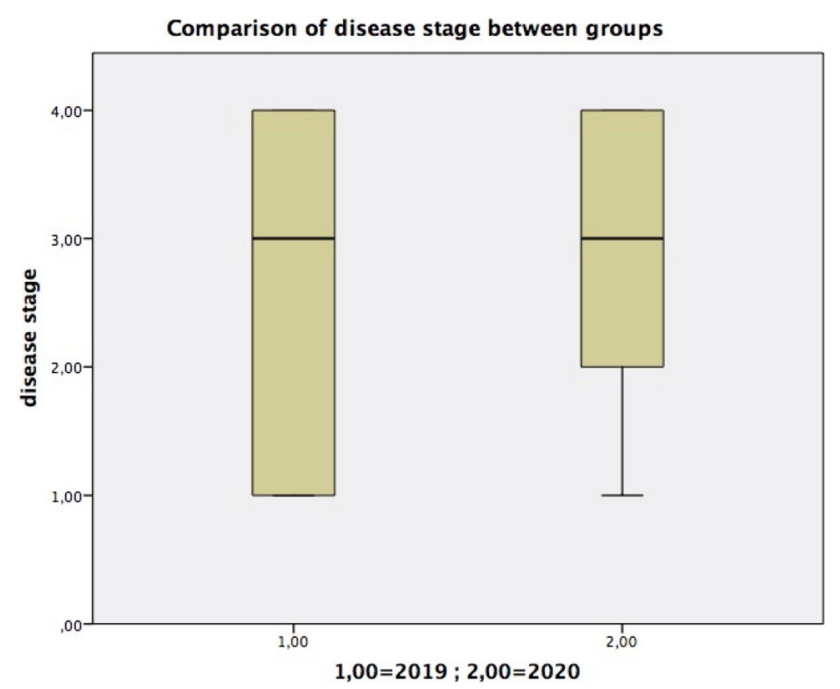

Fig. 2 Comparison of median tumor stage in 2019 and 2020 groups

time periods, no statistically significant differences were appreciated both in 2019 and 2020 groups as depicted in Fig. 3 ( $p>0.05$ for both comparisons).

\section{Discussion}

As a result of the restrictions imposed by the health emergency, an overall reduction in clinical and surgical activities was recorded during the analyzed 2020 period at our institution. This was more considerable for the outpatient first visits and surgical procedures, less for MDT consultations, and more pronounced during the first phase of the pandemic (March-May 2020). Accordingly, a
Table 3 different therapeutic approaches performed in the two groups

\begin{tabular}{lll}
\hline Therapeutic approach & 2019 group & 2020 group \\
\hline Surgery & $33 \%$ & $39.3 \%$ \\
Surgery + radiotherapy & $23.3 \%$ & $25 \%$ \\
Surgery + chemo/radiotherapy & $13.8 \%$ & $6 \%$ \\
Radiotherapy & $4.3 \%$ & $1.8 \%$ \\
Chemo/radiotherapy & $8.6 \%$ & $14.1 \%$ \\
Palliative care & $11.9 \%$ & $10.3 \%$ \\
Abandon of the MDT consultations & $5.1 \%$ & $3.5 \%$ \\
\hline
\end{tabular}

slightly reduced number of patients diagnosed with head and neck cancer was recorded at our institution between March and October 2020, in comparison to 2019.

The most impactful result of our research was the statistically significant difference in the average tumor stage between 2019 and 2020 groups, with a higher average value in the latter. The present data confirm previous hypothesis that the COVID-19 pandemic may have resulted in a more advanced stage at presentation for head and neck cancer [11, 12]. The oncological services should be prepared to potentially treat a higher number of patients with more advanced disease as a result of the COVID-19 outbreak. No statistical differences in baseline characteristics such as age and tumor site were found in the 2019 group versus the 2020 group, nor in the stratification of therapeutic approaches. Interestingly, an increase in the incidence of cancer in advanced stages (IVb-IVc) and in palliative treatments was not appreciated. The long-term 
Fig. 3 distribution of the delay in treatment initiation over the 3 phases in 2020 e 2019

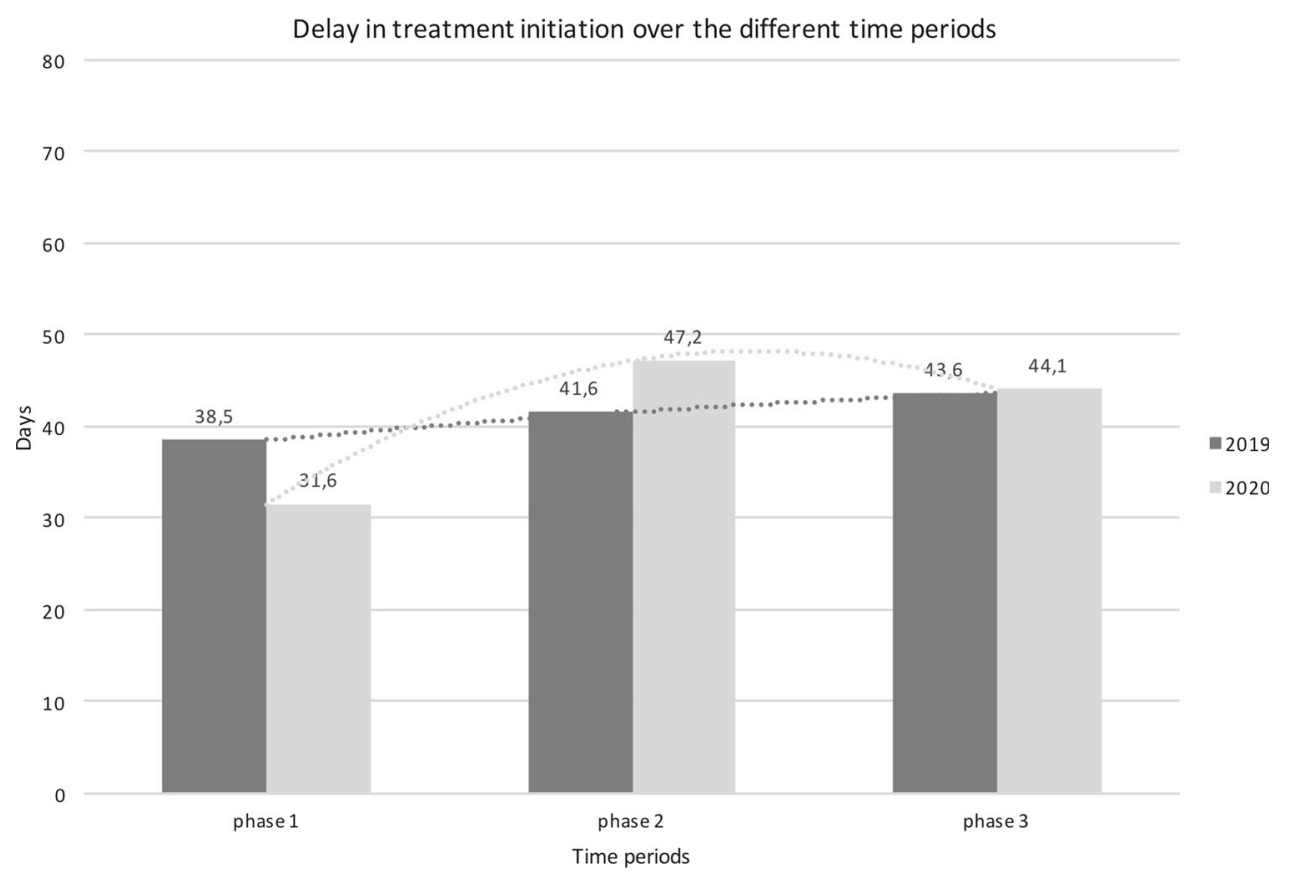

implications in terms of disease-free and overall survival will need quantification in the future [13].

Many reasons support the reduction in provided health care at the basis of such diagnostic delay. Italy has been one of the most affected countries from the beginning of the pandemic. The national health authorities put in place various containment strategies to keep the spread of the virus under control, trying to protect both patients and health-care workers. Especially during the first phase of the pandemic, all hospital admissions for non-urgent reasons were discouraged. The European Society of Surgical Oncology advised against seeing patients older than 70 in the clinics, unless urgent [14]. Also, the travel restrictions and the fear of contagion could have discouraged patients, in particular the elderly and those with pre-existing pathologies, in referring to the healthcare system. All these factors generated a significant reduction in investigations for new cancer diagnoses and cancer surgery. Online consultation and telemedicine [15] have been strongly recommended by oncology organizations (MDAnderson), but it is not always suitable for all patients or all oncology specialists. The COVID-19 pandemic has forced physicians to triage which patients should have access to evaluation urgently versus those who may be delayed. The results of the present study demonstrated the shortcomings of this system.

Other Italian reports testified on the delay in the diagnosis and treatment of tumors during 2020, also in relation to the significant drop in screening exams performed [16, 17]. A telephone survey on patients who had a cancer diagnosis during the COVID-19 pandemic demonstrated that $82.7 \%$ delayed specialist evaluation because of concerns about COVID-19, and those with multiple underlying medical conditions (such as diabetes or hypertension) were significantly more likely to avoid or delay urgent or emergency care compared to people without these conditions [16].

Surgery for HNC was the object of the most severe limitations during the pandemics [18]. This was due to the direct airway manipulations and aerosol-generating procedures, and to the possible need for complex reconstruction, tracheostomy, nasogastric tube placement, tracheotomy, repeated endotracheal tube removal and replacement larynx surgery [19]. HNC surgical teams were considered as highly vulnerable to acquiring COVID-19 during such procedures [20, 21]. Also, reliance on critical care for postoperative recovery is common to many institutions, and this could increase the risk of cross-infection with the SARS-CoV-2 virus. Furthermore, patients who unknowingly have surgery during the incubation period of COVID19 infection appear to be at extremely high risk of morbidity and may have approximately $20 \%$ risk of mortality [22]. It has been estimated that $37 \%$ of cancer surgeries have been cancelled during the COVID-19 pandemic [22]. It is, therefore, probable that patients amenable to surgery were treated at a higher rate with primary radiotherapy or chemo-radiotherapy instead. This aspect was however not statistically confirmed by our results.

A change in strategy was demonstrated by a survey performed on 88 head and neck US surgeons [23]: most respondents were more likely to consider nonsurgical therapy for patients with glottis cancer and for HPV- 
mediated oropharynx cancer as compared to their normal practice. Also, a report by Hanna et al. [6] revealed a doubling of non-surgical treatment indications for same stage cases in the comparison between April-May 2020 and the same period of 2019 .

It has been established that an increase in the time between diagnosis and definitive therapy is associated with decreased overall and disease-free survival [24]. The mean time between diagnostic suspicion and therapy administration in our study was 44 days and it was not statistically different compared to the previous year, contrary to other reports [25]. Most head and neck surgeons, including the authors, advocate for initiation of treatment within 1 month from the diagnosis, and our results allow us to confirm that this protocol was respected also during the pandemics. A retrospective cohort study on $730 \mathrm{HNC}$ patients demonstrated that the optimal time-to-surgery was inferior to 67 days: patients who underwent surgical treatment more than 67 days after diagnosis had a significantly increased risk of death [24]. Although there was an overall restriction in all health services, it is likely that it was counterbalanced by the contextual reduction in routine and non-urgent instrumental and clinical exams, resulting in a substantially unchanged diagnostic process for oncological patients. Also, the need to carefully plan the few available operating sessions (specifically dedicated to oncologic patients) could be responsible for that. Timely intervention, even in the era of COVID-19, is necessary to maintain a high level of care.

\section{Conclusion}

A more advanced stage at presentation was demonstrated at our institution for HNC patients during the first phase of the COVID-19 pandemic in 2020, when compared to the same period in 2019. However, a statistically significant delay in time between diagnosis and therapy administration was not assessed, thus confirming the maintenance of a high level of care even in the COVID-19 era. This knowledge may support treatment decisions both now and during future pandemics.

Funding This research did not receive any specific grant from funding agencies in the public, commercial, or not-for-profit sectors.

\section{Declarations}

Conflict of interest The authors declare that they have no conflict of interest.

Ethics approval This IRB ethical approval is not required at our institution for this type of article.

\section{References}

1. Hamilton W (2020) Cancer diagnostic delay in the COVID-19 era: what happens next? Lancet Oncol 21(8):1000-1002

2. Mireştean CC, Crişan A, Mitrea A, Buzea C, Iancu RI, Iancu DPT (2021) New challenges of treatment for locally advanced Head and Neck cancers in the covid-19 pandemic era. J Clin Med 10(4):587

3. Chan J, Wong EWY, Lam W (2020) Practical aspect of Otolaryngologic clinical services during the 2019 Coronavirus epidemics and experience in Hong Kong. JAMA Otolaryngol Head Neck Surg 146(6):519-520. https://doi.org/10.1001/ jamaoto.2020.0488

4. Bhangu A, Nepogodiev D, Glasbey JC et al (2020) Mortality and pulmonary complications in patients undergoing surgery with perioperative sars-cov-2 infection: an international cohort study. Lancet 396(10243):27-38

5. Vukkadala N, Qian ZJ, Holsinger FC, Patel ZM, Rosenthal E (2020) COVID-19 and the Otolaryngologist: preliminary evidence-based review. Laryngoscope 130(11):2537-2543

6. Hanna TP, Evans GA, Booth CM (2020) Cancer, COVID-19 and the precautionary principle: prioritizing treatment during a global pandemic. Nat Rev Clin Oncol 17(5):268-270

7. American College of Surgeons. Covid-19: Guidance for triage of non-emergent surgical procedures

8. American Academy of Otolaryngology - Head and Neck surgery. Otolaryngologists and the COVID-19 Pandemic

9. Tevetoğlu F, Kara S, Aliyeva C, Yıldırım R, Yener HM (2021) Delayed presentation of head and neck cancer patients during COVID-19 pandemic. Eur Arch Oto-Rhino-Laryngol 0123456789:1-5

10. Matos LL, Forster CHQ, Marta GN et al (2021) The hidden curve behind COVID-19 outbreak: the impact of delay in treatment initiation in cancer patients and how to mitigate the additional risk of dying - the head and neck cancer model. Cancer Causes Control 32(5):459-471

11. Kiong KL, Diaz EM, Gross ND, Diaz EM, Hanna EY (2021) The impact of COVID-19 on head and neck cancer diagnosis and disease extent. Head Neck 43(6):1890-1897

12. Galli J, Settimi S, Tricarico L, Almadori G, Paludetti G (2020) Clinical and surgical management of patients with head and neck cancer in a COVID-19 dedicated center in Italy. Head Neck 42(7): $1466-1470$

13. Wise J (2020) Covid-19: Cancer mortality could rise at least $20 \%$ because of pandemic, study finds. BMJ 369:m1735

14. Burki TK (2020) Cancer guidelines during the COVID-19 pandemic. Lancet Oncol 21(5):629-630

15. Banerjee S, Voliotidis D, Parvin L, Rama SPK (2021) Telephone triage of suspected head and neck cancer patients during the coronavirus disease 2019 pandemic using the Head and Neck cancer risk calculator version 2. J Laryngol Otol 135(3):241-245

16. De Luca P, Bisogno A, Colacurcio V et al (2020) Diagnosis and treatment delay of head and neck cancers during COVID-19 era in a tertiary care academic hospital: what should we expect? Eur Arch Oto-Rhino-Laryngol 2021:1-5

17. Chiaravalli S, Ferrari A, Sironi G et al (2020) A collateral effect of the COVID-19 pandemic: delayed diagnosis in pediatric solid tumors. Pediatr Blood Cancer 67(10):2-3

18. Crosby DL, Sharma A (2020) Evidence-based guidelines for management of Head and Neck Mucosal Malignancies during the COVID-19 Pandemic. Otolaryngol - Head Neck Surg 163(1):16-24

19. Mick P, Murphy R (2020) Aerosol-generating otolaryngology procedures and the need for enhanced PPE during the COVID-19 
pandemic: a literature review. J Otolaryngol - Head Neck Surg 49(1): $1-10$

20. Day AT, Sher DJ, Lee RC et al (2020) Head and neck oncology during the COVID-19 pandemic: reconsidering traditional treatment paradigms in light of new surgical and other multilevel risks. Oral Oncol 105:104684

21. Tang JW, Marr LC, Li Y, Dancer SJ (2021) Covid-19 has redefined airborne transmission. BMJ 373(April):1-2

22. Shaw R, Schache AG, Ho MWS et al (2021) UK Head and neck cancer surgical capacity during the second wave of the COVID19 pandemic: Have we learned the lessons? COVIDSurg collaborative. Clin Otolaryngol 46(4):729-735

23. Brody RM, Albergotti WG, Shimunov D et al (2020) Changes in head and neck oncologic practice during the COVID-19 pandemic. Head Neck 42(7):1448-1453
24. Rygalski CJ, Zhao S, Eskander A et al (2021) Time to surgery and survival in Head and Neck Cancer. Ann Surg Oncol 28(2):877-885

25. Arduino PG, Conrotto D, Broccoletti R (2021) The outbreak of Novel Coronavirus disease (COVID-19) caused a worrying delay in the diagnosis of oral cancer in north-west Italy: the Turin Metropolitan area experience. Oral Dis 27(S3):742-743

Publisher's Note Springer Nature remains neutral with regard to jurisdictional claims in published maps and institutional affiliations. 